\title{
THE BLOCK NUMERICAL RANGE OF ANALYTIC OPERATOR FUNCTIONS
}

\author{
Agnes Radl, Christiane Tretter and Markus Wagenhofer
}

\begin{abstract}
We introduce the block numerical range $W^{n}(\mathscr{L})$ of an operator function $\mathscr{L}$ with respect to a decomposition $H=H_{1} \oplus \ldots \oplus H_{n}$ of the underlying Hilbert space. Our main results include the spectral inclusion property and estimates of the norm of the resolvent for analytic $\mathscr{L}$. They generalise, and improve, the corresponding results for the numerical range (which is the case $n=1$ ) since the block numerical range is contained in, and may be much smaller than, the usual numerical range. We show that refinements of the decomposition entail inclusions between the corresponding block numerical ranges and that the block numerical range of the operator matrix function $\mathscr{L}$ contains those of its principal subminors. For the special case of operator polynomials, we investigate the boundedness of $W^{n}(\mathscr{L})$ and we prove a Perron-Frobenius type result for the block numerical radius of monic operator polynomials with coefficients that are positive in Hilbert lattice sense.
\end{abstract}

Mathematics subject classification (2010): 47A56, 47A12.

Keywords and phrases: Numerical range, operator function, spectrum, eigenvalue, resolvent, block numerical radius, positive operator, Perron-Frobenius theorem, gyroscopic system.

\section{REFERENCES}

[1] T. Betcke, N. J. Higham, V. Mehrmann, C. Schröder, F. Tisseur, NLEVP: a collection of nonlinear eigenvalue problems, ACM Trans. Math. Software, 39 (2): Art. 7, 28, 2013.

[2] F. F. BONSALL, Endomorphisms of partially ordered vector spaces; Endomorphisms of a partially ordered vector space without order unit, J. London Math. Soc., 30: 144-153, 1955.

[3] G. Frobenius, Über Matrizen aus nicht negativen Elementen, Sitzungsber. Königl. Preuss. Akad. Wiss., Berlin, 456-477, 1912.

[4] K. E. Gustafson And D. K. M. RaO, Numerical Range, Universitext, Springer-Verlag, New York, 1997, The field of values of linear operators and matrices.

[5] P. R. Halmos, A Hilbert Space Problem Book, volume 19 of Graduate Texts in Mathematics, Springer-Verlag, New York, second edition, 1982, Encyclopedia od Mathematics and its Applications, 17.

[6] D. Hinrichsen And A. J. Pritchard, Mathematical systems theory, I, volume 48 of Texts in Applied Mathematics, Springer-Verlag, Berlin, 2005, Modelling, state space analysis, stability and robustness.

[7] T. Kato, Perturbation theory for linear operators, Classics in Mathematics, Springer-Verlag, Berlin, 1995, Reprint of the 1980 edition.

[8] M. KREÎN, Sur les opérations linéaires transformant un certain ensemble conique en lui-même, C. R. (Doklady) Acad. Sci. URSS (N.S.), 23: 749-752, 1939.

[9] M. G. KREǏN, M. A. RUTMAN, Linear operators leaving invariant a cone in a Banach space, Uspehi Matem. Nauk (N. S.), Vol. 3, 1 (23): 3-95, 1948.

[10] H. LANGER, Über stark gedämpfte Scharen im Hilbertraum, J. Math. Mech., 17: 685-705, 1967/1968.

[11] H. Langer, A. S. Markus, V. I. Matsaev, And C. TRetter, A new concept for block operator matrices: the quadratic numerical range, Linear Algebra Appl., 330 (1-3): 89-112, 2001.

[12] P. LANCASTER AND P. J. PSARRAKOS, The numerical range of self-adjoint quadratic matrix polynomials, SIAM J. Matrix Anal. Appl., 23 (3): 615-631 (electronic), 2001/02. 
[13] C.-K. Li And L. Rodman, Numerical range of matrix polynomials, SIAM J. Matrix Anal. Appl., 15 (4): 1256-1265, 1994.

[14] H. Langer, A. S. Markus, C. Tretter, Corners of numerical ranges, Oper. Theory Adv. Appl., 124: 385-400, Birkhäuser, Basel, 2001.

[15] H. LANGER AND C. TRETTER, Spectral decomposition of some nonselfadjoint block operator matrices, J. Operator Theory, 39 (2), 339-359, 1998.

[16] G. Maibaum, Über Scharen positiver Operatoren, Math. Ann., 184: 238-256, 1969/1970.

[17] A. I. MARKuSheVICH, Theory of functions of a complex variable, Vol. I, II, III, Chelsea Publishing Co., New York, english edition, 1977. Translated and edited by Richard A. Silverman.

[18] A. S. MARKUS, Introduction to the spectral theory of polynomial operator pencils, volume 71 of Translations of Mathematical Monographs, American Mathematical Society, Providence, RI, 1988.

[19] A. S. MARKUS AND V. I. MATSAEV, Some estimates for the resolvent and for the lengths of Jordan chains of an analytic operator function, In recent advances in operator theory (Groningen, 1998), volume 124 of Oper. Theory Adv. Appl., pages 473-479, Birkhäuser, Basel, 2001.

[20] P. Meyer-Nieberg, Banach Lattices, Universitext, Springer-Verlag, Berlin, 1991.

[21] P. C. MüLlER, Stabilität und Matrizen, Springer-Verlag, Berlin, 1977. Matrizenverfahren in der Stabilitätstheorie linearer dynamischer Systeme, Ingenieurwissenschaftliche Bibliothek/Engineering Science Library.

[22] P. J. PsarRakos AND M. J. Ts atsomeros, A primer of Perron-Frobenius theory for matrix polynomials, Linear Algebra Appl., 393: 333-351, 2004.

[23] O. Perron, Zur Theorie der Matrices, Math. Ann., 64 (2): 248-263, 1907.

[24] A. RADL, The block numerical range of positive operators on Hilbert lattices, Integral Equ. Oper. Theory, 75: 459-472, 2013 DOI:10.1007/s00020-013-2032-x.

[25] A. Radl, C. TRetter, M. WAgenhofer, Corners of the block numerical range of analytic operator functions, Preprint, 16 pp., 2013.

[26] R. T. RAU, On the peripheral spectrum of monic operator polynomials with positive coefficients, Integral Equ. Oper. Theory, 15 (3): 479-495, 1992.

[27] H. H. Schaefer, Banach Lattices and Positive Operators, Springer-Verlag, New York, 1974, Die Grundlehren der mathematischen Wissenschaften, Band 215.

[28] H. H. SchaEfER, Some spectral properties of positive linear operators, Pacific J. Math., 10: 10091019, 1960.

[29] G. Schweitzer, W. Schichten, P. C. Müller, W. Hübner, J. LÜCkel, G. Sandweg And R. LAUTENSCHLAGER, Kreiselverhalten eines elastisch gelagerten Rotors, Ingenieur Archiv, 41 (2): $110-140,1972$.

[30] F. Tisseur AND K. MeErbergen, The quadratic eigenvalue problem, SIAM Rev., 43 (2): 235-286, 2001.

[31] C. TRetTER, Spectral Theory of Block Operator Matrices and Applications, Imperial College Press, London, 2008.

[32] C. TRETTER, The quadratic numerical range of an analytic operator function, Complex Anal. Oper. Theory, 4 (2): 449-469, 2010.

[33] C. TRETTER AND M. WAGENHOFER, The block numerical range of an $n \times n$ block operator matrix, SIAM J. Matrix Anal. Appl., 24 (4): 1003-1017 (electronic), 2003.

[34] M. WAgenhofer, Der blocknumerische Wertebereich, 2000. Diploma Thesis - Universität Regensburg.

[35] M. WAGENHOFER, $C++$-code for calculating numerical ranges, quadratic numerical ranges, and block numerical ranges of analytic operator functions, Private communication, 2007.

[36] M. WAGENHOFER, The block numerical range of an $n \times n$ block operator matrix, Thesis (Ph.D.), 88 pp. Universität Bremen, 2007. 